Recepción: 14/ 08 / 2018

Aceptación: 21 / $10 / 2018$

Publicación: 03 / 12 / 2018

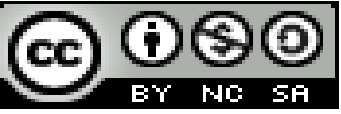

Ciencias de la salud

Artículo de revisión

\title{
Algunas consideraciones acerca de los pernos de fibra de vidrio
}

\author{
Some considerations about glass fiber bolts
}

Algumas considerações sobre os parafusos de fibra de vidro

Alex X. Bravo-Rodríguez ${ }^{\mathrm{I}}$

alex.bravor@ug.edu.ec

Marisela S. Villarreal-Salazar II

marisela.vs@ug.edu.ec

Vanessa J. Veintimilla-Abril III

vanessa.veintimillaab@ug.edu.ec

\section{Correspondencia: alex.bravor@ug.edu.ec}

\footnotetext{
${ }^{\text {I }}$ Magíster en Gerencia de Salud para el Desarrollo Local, Especializado en Dentística Restauradora, Odontólogo, Docente de la Universidad de Guayaquil, Guayaquil, Ecuador.

II Especialista en Rehabilitación Oral, Odontóloga, Docente de la Universidad de Guayaquil, Guayaquil, Ecuador.

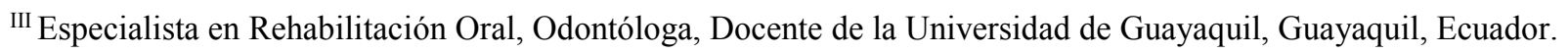




\section{Resumen}

El diente tratado endodónticamente y su rehabilitación, sigue siendo uno de los grandes paradigmas de la odontología contemporánea. A tales efectos se realizó una revisión bibliográfica exhaustiva para exponer algunos aspectos relacionados con esta temática (finalidad de los pernos, funciones, composición de los pernos de fibra de vidrio, entre otros) y se puntualizó en resultados de investigaciones con respecto a pruebas de adhesión en pernos de fibra de vidrio.

Palabras clave: pernos de fibra de vidrio; conducto radicular; endodoncia.

\section{Abstract}

The tooth treated endodontically and its rehabilitation, remains one of the great paradigms of contemporary dentistry. To this end, an exhaustive bibliographic review was carried out to expose some aspects related to this subject (purpose of bolts, functions, composition of glass fiber bolts, among others) and it was specified in research results with respect to adhesion tests in fiberglass bolts.

Key words: fiberglass bolts; root canal; endodontics.

\section{Resumo}

$\mathrm{O}$ dente tratado endodonticamente e sua reabilitação, continua sendo um dos grandes paradigmas da odontologia contemporânea. Para tanto, foi realizada uma revisão bibliográfica exaustiva para expor alguns aspectos relacionados a esse assunto (propósito de parafusos, funções, composição de parafusos de fibra de vidro, entre outros) e foi especificado em resultados de pesquisas com respeito a testes de aderência. Em parafusos de fibra de vidro.

Palavras chave: parafusos de fibra de vidro; canal radicular; endodontia.

\section{Introducción}

La restauración de los dientes endodonciados ha sido un tema ampliamente estudiado en la literatura científica. En la primera década del siglo XXI comienzan a florecer los materiales adhesivos a base de resina, con buenos resultados clínicos, no había aún un consenso definido a la hora de restaurar los dientes endodonciados. (Mannocci F, Bhuva B, Stern S 2008) 
Marcé Clavillé (2015) informa que la aparición de nuevos diseños anatómicos, materiales de los pernos intrarradiculares y los sistemas adhesivos actuales, han hecho que los protocolos de restauración de los dientes endodonciados, se hayan modificado, para ser más conservadores y sólo aquellos dientes con poca estructura dental remanente requerirán de un poste intrarradicular para ser restaurados satisfactoriamente.

En el marco de las observaciones anteriores, según Kurer (1992) expone que contrariamente a lo que se pensaba en tiempos pasados, los pernos no se colocan para reforzar las raíces, sino más bien para mantener el muñón adherido a la raíz, y sobre el mismo colocar de manera segura y firme una corona que pueda restituir la función y apariencia al diente. (Arregui Valdivieso K. 2011)

Disimiles investigaciones han comprobado que el perno no da fuerza al diente; de hecho, puede debilitarlo, si se ha hecho demasiado grueso o largo. La decisión sobre el uso de un perno en un diente tratado endodónticamente, comienza con el entendimiento del verdadero propósito del perno. (Arregui Valdivieso K. 2011)

La razón principal para el uso de un perno, es la de conectar la estructura radicular al núcleo. No tiene como propósito "reforzar" al diente. (Arregui Valdivieso K. 2011)

Las pautas para la utilización de los pernos prefabricados son variables; los pernos prefabricados pueden ser tan fuertes, o más fuertes, que los pernos y núcleos colados; las aleaciones de titanio y acero inoxidables son las más utilizadas para pernos. (Arregui Valdivieso K. 2011)

Sin lugar a duda el factor de éxito más importante en pernos, es la cantidad de estructura dental coronaria remanente. $2 \mathrm{~mm}$ de cuello remanente en el diente; un perno puede no ser necesario si hay más de estructura coronal remanente, y si la oclusión no es muy fuerte. (Arregui Valdivieso K. 2011)

Entre las funciones de los pernos se pueden señalar:

Retener la reconstrucción coronaria.

Distribuir las fuerzas en el área radicular evitando su concentración en el área coronaria. 
Trasladar la superficie de soporte a zonas de contacto con el hueso alveolar. (Arregui Valdivieso K. 2011)

Reseña Monticelli F (2006) que es de suma importancia la restauración del diente endodonciado para la funcionalidad del sistema estomatognático; una alternativa de tratamiento son los pernos de fibra de vidrio.

En la década de los noventas, surgen los pernos intrarradiculares de fibra de vidrio, los cuales presentan excelentes propiedades y características. Nuevas investigaciones deben ser realizadas para despejar cualquier duda, analizando la longevidad de los pernos intrarradiculares de fibra de vidrio. (Da Cunha Uchôa R, 2008)

Se acopia en la literatura que en varios momentos se ha investigado los procedimientos del tratamiento de superficie en pernos de fibra de vidrio, los cuales son mecánicos, químicos o la combinación de ambos, encontrando en los primeros la aplicación del peróxido de hidrógeno, silano, ácido fosfórico, entre otros. (Domínguez S 2017)

Observando la literatura, expresa que los pernos de fibra de vidrio son corrientemente utilizados en la actualidad en el tratamiento post endodóntico de piezas dentarias con insuficiente estructura coronaria, teniendo como alguno de sus objetivos formar un solo núcleo en conjunto con la pieza dentaria tratada. Por tanto, es importante la calidad de unión establecida entre la superficie del poste y el muñón. (Resende V, De Sousa M, Guimarães M, E C, Soares C 2014)

\section{Composición y propiedades de los pernos de fibra de vidrio.}

Los PPF normalmente se componen de finísimas fibras unidireccionales pretensadas de Carbono (C), Vidrio o Cuarzo, en general conglomeradas con una resina del tipo Epoxi a la que se puede añadir resina de Bis-GMA (de mayor afinidad con los cementos resinosos) o incluso en algún caso, ser totalmente en base a dimetacrilatos. Esta combinación de elementos proporciona elasticidad comparable a la de los tejidos dentinarios - entre 18 y 24 Giga Pascal (GPa) junto con adecuadas cualidades mecánicas. La proporción de fibras incorporada está en relación directa con su resistencia mecánica y su ME. Los postes de C presentan promedialmente un ME de 34, los de FV 28 y los de Cuarzo 24. Entre los metales (siempre alejados) los que mejor se comportan son el Oro (Au) dental con un módulo de 80 y el Titanio (Ti) con uno de 110 GPa. El Acero 
Inoxidable (A. Inox.), el Níquel-Cromo (Ni-Cr) y los prefabricados Cerámicos (Circonio) son extremadamente rígidos, presentando cifras de alrededor de $200 \mathrm{GPa}$ de ME. De ahí se deduciría que los pernos rígidos metálicos y cerámicos producirían graves fracturas longitudinales, denominadas "catastróficas" por ser en su mayoría intratables. (Calabria Díaz, Hugo. 2010)

En los pernos fibroadheridos se ha informado la casi imposibilidad de producir fracturas radiculares "in vitro" o la menor frecuencia y gravedad de las mismas. Clínicamente el descementado y la rotura del propio perno serían las causas de fracaso más frecuentes, seguidos por la falla en el muñón coronario. La fractura radicular sería un hecho raro o escaso. (Calabria Díaz, Hugo. 2010)

Sus propiedades anisotrópicas indican que con una angulación de fuerzas oblicuas para valores de carga en función normal, se obtendrían cifras aproximadas a $21 \mathrm{GPa}$, muy favorables para la disipación de tensiones. (Rijk W.2000)

Los pernos pueden ser cónicos simples o de doble conicidad, cilíndricos o cilindrocónicos; cabe agregar que los cónicos buscan acompañar la forma del diente, ahorrando tejido, aunque perdiendo algo en capacidad retentiva. Se los responsabiliza de transmitir esfuerzos en cuña, cosa que no sucedería con los cilíndricos, de mayor capacidad retentiva. En estos últimos la profundización apical puede ocasionar debilitamiento en las paredes radiculares. (Rijk W.2000)

Al referirse a la facilidad de remoción y retratamiento son fácilmente removibles por medio del fresado, lo que se presenta como una de sus cualidades más ventajosas. Existen avíos ofrecidos al respecto, aunque también se puede usar una fresa "Largo" de conducto, luego de iniciar el camino con fresa redonda pequeña (serán guiadas por la disposición longitudinal de las fibras). (Rijk W.2000).

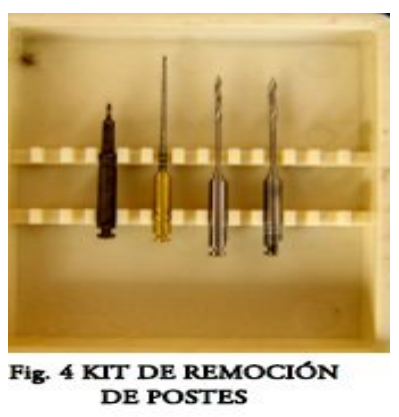


En relación a su radiolucidez, su composición no es favorable para el control radiográfico por lo cual se han agregado opacificadores (FC Postec, Ivoclar-Vivadent) y se usan cementos opacificados que denuncian su silueta (Duo LinK, Bisco). Una fina espiga metálica se presenta en Reforpost RX de Angelus para tales fines. (Nocchi E C. 2008)

Cada día es mayor el interés de nuestros pacientes en mejorar la apariencia de sus sonrisas y con ello lograr una mayor confianza en la comunicación con sus semejantes. Esto se debe a la influencia de los medios de comunicación social, que han impuesto patrones que generan cambios en la conciencia estética de las personas, donde se identifica el éxito personal, con aquellos individuos que presentan una sonrisa bella y placentera. Esto ha obligado a la odontología a buscar, a través de sus procedimientos y materiales, la estética; el cambio en la filosofía terapéutica de la Odontología restauradora moderna, basada en la mínima intervención y máxima conservación de los tejidos dentarios, ha provocado una transformación importante en la rehabilitación funcional y estética del diente despulpado. Un gran porcentaje de piezas dentarias despulpadas son restauradas hoy, sin la necesidad de realizar un anclaje radicular, por otro lado los pernos de fibra de vidrio por sus cualidades mecánicas y estéticas han ganado terreno, desplazando en muchos casos a los pernos metálicos. Los pernos de fibra de vidrio han sido blanco de investigaciones constantes para unir funcionalidad con estético, (González Blanco O, Solórzano Peláez A, Balda Zavarce R. 1999), (Pignata Volpe, Sergio, Vola Gelmini, Joanna, \& Buchtik Efimenco, Natalí. 2012).

Cualidades favorables de los pernos de fibra de vidrio:

No estresantes.

\section{Estéticos.}

No corroibles.

De fácil remoción.

Costo razonable.

Sellado endodóntico complementado.

Menor número de sesiones. 
Posibilidad de cementado adhesivo.

Afinidad estructural poste-cementos.

Posibilidad de transmisión de luz. (Calabria Díaz, Hugo. 2010).

\section{Cualidades desfavorables:}

Posibilidad de descementado.

Posibilidad de fractura del muñón

Posibilidad de fractura del perno.

Cementado adhesivo con interrogantes.

Conformación dificultosa del muñón coronario.

Diámetros y formas no anatómicas (no es universal)

Excesiva flexibilidad (descementado, microfiltración). (Calabria Díaz, Hugo. 2010).

\section{Técnica (Procedimientos clínicos):}

Saravia Rojas, Á (2007) mantiene que existen varias técnicas descritas para restaurar órganos dentales para quienes recibieron tratamiento de endodoncia en conductos amplios, lo que es de vital importancia en conductos radiculares en forma elíptica como los caninos, premolares mandibulares o en casos en los que el conducto resulta excesivamente amplio, debido al proceso carioso o en órganos dentales permanentes jóvenes dependientes de su propia anatomía.

El protocolo para llevar a cabo esta técnica, consiste primero en desobturar el sellado provisional del conducto con fresas Gates o Peeso, seguidamente se prueba en el conducto el perno de fibra de vidrio seleccionado observándose que este oscile en sentido mesio - distal y vestíbulo palatino/ lingual, después se comprueba que el conducto esté liso y homogéneo para que no generan atrapamiento de la resina compuesta durante el modelado del conducto.

El perno seleccionado es desinfectado con hipoclorito al $5 \%$ por 2 minutos, se lava con suero fisiológico y se seca con una gasa o con aire. Luego se aplica silano sobre la superficie del espigo y se espera entre 1 a 2 minutos. Después, se coloca un sistema adhesivo usando el microbrush 
sobre toda la superficie del perno. Se aplica aire por 1-5 segundos y se fotocura usando una unidad de fotopolimerizado de acuerdo al tiempo sugerido por el fabricante.

Para la confección del modelado anatómico se requiere una resina compuesta que tenga poca contracción de polimerización pudiendo ser del tipo microhíbrida, nanohíbrida o de nanotecnología. Se dispensa alrededor del perno una gran porción, que luego será insertada en conjunto con el poste al conducto de la pieza. Esta inserción debe ser realizada con movimientos oscilantes, el exceso de material es removido con una espátula, el material restante a nivel cervical es usado para modelar el muñón. Realizados estos pasos, se hace un fotocurado inicial de 5 segundos, luego se retira el poste con un movimiento de tracción y ya fuera se complementa la fotopolimerización. El perno anatómico es arenado con óxido de aluminio de 25 micras, para generar una deformación plástica permanente en la superficie del mismo, obteniendo una superficie porosa para incrementar la retención micromecánica. Se aplica el silano en la superficie del perno anatómico y se espera de 1 a 2 minutos, posteriormente se aplica el agente adhesivo y se le aplica aire de 1 a 5 segundos, pero no se fotopolimeriza, ya que de hacerlo ocuparía un espacio mayor en el conducto en conjunto con el adhesivo aplicado al diente y el cemento resinoso. Se realizan los procedimientos convencionales de adhesión en el sustrato dentario (acondicionamiento ácido, lavado del ácido y aplicación del adhesivo), teniendo en cuenta que tanto el adhesivo como el cemento deben ser de curado dual. Se aplica el cemento con un léntulo, dispensando homogéneamente el cemento en las paredes del conducto, se introduce el poste con movimientos oscilantes para evitar la formación de brechas o burbujas en la interfaz cemento - adhesivo. Y finalmente se fotopolimeriza, de acuerdo al tiempo sugerido, en esta última fotopolimerización serán activados el adhesivo de la superficie del poste, el cemento resinoso y el adhesivo de la dentina radicular, mediado por la capacidad de transmisión de luz de los pernos de fibra de vidrio.

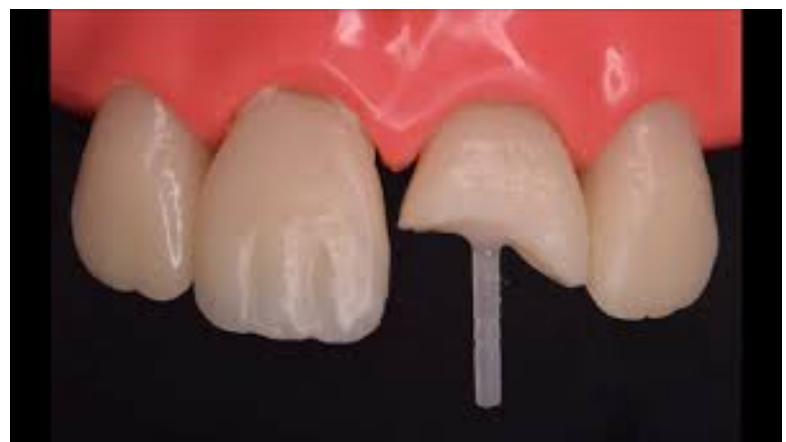




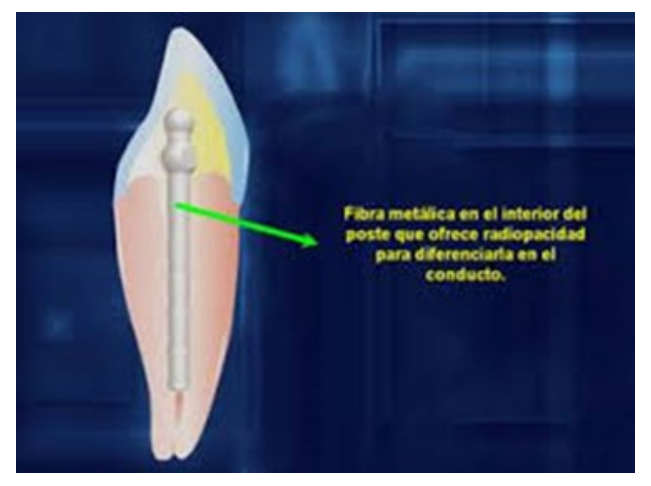

Entre las precauciones a tener en cuenta se podrían nombrar: conducto expulsivo, inadaptación anatómica y fuerzas extremas. (Calabria Díaz, Hugo. 2010).

Resulta oportuno aclarar que no se debe indicar los pernos de fibras de vidrio cuando existe, discrepancia grave en el eje corona-raíz, discrepancia importante con la anatomía radicular y Nulo remanente coronario. (Calabria Díaz, Hugo. 2010)

Revelan datos de investigaciones realizadas por Robles Romero D M (2012), acerca de pruebas de adhesión en pernos de fibra de vidrio utilizando dos diferentes cementos a base de resina. La prueba de adhesión reportó que los postes cementados con Maxcem requieren de menor fuerza para ser desalojados del conducto radicular. Existiendo una diferencia significativa entre los dos grupos. Con respecto al tipo de falla observada la prevalencia de falla tipo adhesivo (cementoporne y porne-dentina). Así mismo resulto el peróxido de hidrógeno al 9\%(30 volúmenes, 10 min)+silano (60s) la sustancia con mayor resistencia adhesiva entre el poste de fibra de vidrio y el muñón en estudio realizado por Domínguez S. (2015). Otros autores referidos a este mismo aspecto plantearon en su investigación que la técnica de irrigación con $\mathrm{NaClO}$ al 5.25\% demostró ser la que menor interferencia produjo en la interfase $\mathrm{CD} / \mathrm{D}$ y $\mathrm{CD} / \mathrm{P}$ presentando el mayor porcentaje de adhesión tanto en la interfase $\mathrm{CD} / \mathrm{D}$ y cohesión en la interfase $\mathrm{CD} / \mathrm{P}$.(Alava Freire 2012)

Entre otras investigaciones tenemos la realizada por Ortiz Naranjo (2010) el cual concluye que los diferentes estudios indican que los pernos de fibra poseen un módulo de elasticidad similar a la dentina, evitando que los dientes con tratamiento endodóntico se fracturen. 
A manera de colofón, el éxito de la restauración es primordial efectuar una apropiada selección del tipo de perno, eliminar la menor cantidad posible de estructura dentaria, conseguir un buen efecto férula y conseguir una buena adhesión del perno en caso de que éste sea de fibra de vidrio.

\section{Referencias Bibliográficas}

Arregui Valdivieso K, (2011). Estudio comparativo entre postes colados y prefabricados en las restauraciones dentales fijas" Recuperado

de http://repositorio.ug.edu.ec/bitstream/redug/7263/1/ARREGUIkarina.pdf

Alava Freire M, Mena Córdova N, Sandoval Vernimmen. (2012). Evaluación de la interfase de adhesión-cohesión entre el poste de fibra de vidrio, cemento dual y dentina, previa irrigación con 2 sustancias desinfectantes. Revista odontología mexicana. 16(3), Recuperado de http://www.medigraphic.com/pdfs/odon/uo-2012/uo123e.pdf

Nocchi E C. (2008). Odontología restauradora. Salud y estética. Edit. Med. Panamericana $2^{\circ}$ Edicción, 2008

Calabria Díaz, Hugo. (2010). Postes prefabricados de fibra: Consideraciones para su uso clínico. Odontoestomatología, 12 (Supl. 16), 4-22. Recuperado de http://www.scielo.edu.uy/scielo.php?script=sci_arttext\&pid=S1688$93392010000300002 \& \operatorname{lng}=$ es\&tlng=es.

Da Cunha Uchôa R, 2008. Hipólito Paredes A, Pereira de Melo A, Viégas Montenegro R, Pedrosa R, Braz R. (2014). Pernos intrarradiculares de fibra de vidrio: caso clínico. Acta odontológica Venezolana. 46(4).

Domínguez S, Castillo Andamayo, D, Ramos O, Rozas A. (2017). Evaluación de la resistencia adhesiva entre el poste de fibra de vidrio y el muñón de resina utilizando diferentes tratamientos de superficie Revista Estomatológica Herediana, 27 (3), 2017, pp. 153-162 Universidad Peruana Cayetano Heredia Lima, Perú.

González Blanco O, Solórzano Peláez A, Balda Zavarce R. (1999). Estética en odontología. Parte i aspectos psicológicos relacionados a la estética bucal. Recuperado de 
https://www.actaodontologica.com/ediciones/1999/3/estetica_odontologia_aspectos_psicologicos .asp

Mannocci f, Bhuva B, Stern S. Restoring teeth following root canal retreatment. Endodontic Topics 2008; 19 (1):125- 152.

Marcé Clavillé M. Estrategias adhesivas de los postes de fibra de vidrio. (2015). Recuperado de https://www.tdx.cat/bitstream/handle/10803/321361/Tesi_Marta_Marc\%C3\%A9_Clavill\%C3\% A9.pdf? sequence $=1 \&$ isAllowed $=\mathrm{y}$

Monticelli F, Toledano M, Tay F, Cury A, Goracci C Ferrari M. (2006). Post-surface conditioning improves interfacial adhesion in post/core restorations. Dental Materials; 22: 602-9.

Ortiz Naranjo L (2010). Postes de Fibra. Recuperado de http://www.cop.org.pe/bib/tesis/LORENACECILIAORTIZNARANJO.pdf

Pignata Volpe, Sergio, Vola Gelmini, Joanna, \& Buchtik Efimenco, Natalí. (2012). Técnica del Poste Anatómico (Grandini): Caso clínico. Odontoestomatología, 14(19), 4-13. Recuperado de http://www.scielo.edu.uy/scielo.php?script=sci_arttext\&pid=S1688-

93392012000100002\&lng=es\&tlng=es.

Robles Romero D M, Sánchez Huerta H A, Rodríguez Arámbula J,* Ríos Álvarez S. (2012). Pruebas de adhesión en postes de fibra de vidrio utilizando dos diferentes cementos a base de resina. Recuperado de http://www.uan.edu.mx/d/a/publicaciones/revista_tame/numero_1/Tam121-02.pdf

Rijk W. Removal of fiber posts from endodontically treated teeth. Am J Dent 2000; 13:19B-21B.

Resende V M, De Sousa M, Guimarães M, C, Soares C. (2014). Effect of surface treatment of fiber glass post on bond strength to root dentin. Braz

Dent J; 25(4): 314-20.

Saravia Rojas, Miguel Ángel. (2007). Postes Anatómicos Completamente Estéticos. Revista Fórmula Odontológica. 5 (1), 